\title{
Eficácia antitussígena de duas formulações fitoterápicas
}

\author{
[Antitussive efficacy of two phytotherapics formulations] \\ F.B. Mello ${ }^{1}$, J.R.B. Mello ${ }^{2 *}$ \\ ${ }^{1}$ Aluna de pós-graduação - ICBS-UFRGS \\ ${ }^{2}$ Departamento de Farmacologia - ICBS - UFRGS \\ Av. Bento Gonçalves, 9090 - Bairro Agronomia \\ 91540-000 - Porto Alegre, RS
}

\begin{abstract}
RESUMO
Avaliaram-se os efeitos antitussígenos-expectorantes de duas formulações fitoterápicas utilizando-se três modelos biológicos diferentes. Foram utilizados ratos Wistar no modelo da secreção das vias aéreas, cobaias no modelo de tosse induzido por ácido cítrico e codornas japonesas na determinação da velocidade de transporte mucociliar. Os animais foram distribuídos em grupos e tratados por via oral com as formulações, com doses equivalentes a 10 vezes a terapêutica recomendada, $9 \mathrm{ml}_{\mathrm{kg}} \mathrm{kg}^{-1}$. O grupo-controle negativo de cada espécie foi tratado com solução fisiológica, $10 \mathrm{ml}_{\mathrm{kg}} \mathrm{kg}^{-1} \mathrm{O}$ grupo-controle positivo no modelo de tosse induzida pelo ácido cítrico foi tratado com morfina, $1 \mathrm{mg} \cdot \mathrm{kg}^{-1}$, por via subcutânea. No modelo em que foram utilizados ratos e codornas, o grupo-controle positivo recebeu erdosteína por via oral, $600 \mathrm{mg} \cdot \mathrm{kg}^{-1}$. Os resultados mostraram que as duas formulações fitoterápicas foram eficazes no reflexo da tosse em cobaias, causando $36,4 \%$ e $27,3 \%$, respectivamente, de redução. Nos modelos de secreção das vias aéreas e determinação da velocidade de transporte mucociliar, ambas as formulações não apresentaram eficácia significativa.
\end{abstract}

Palavras-chave: antitussígeno, expectorante, fitoterapia, Gripalplus Solução®, Melagrião®

\section{ABSTRACT}

The antitussive-expectorant effects of two phytotherapic formulations available in the Brazilian market were evaluated using three different biological models. Each phytotherapic formulation, with different composition, had the same batch number and fabrication date. The trade names of the phytotherapics were: Gripalplus Solução ${ }^{\circledR}$ and Melagrião $\AA$. Wistar rats were used in the airway secretion model, guinea pigs in the citric acidinduced cough model and japanese quails in the mucociliary transport rate determination. The animals (one group/formulation) were divided in the two phytotherapic groups and orally treated with the equivalent to ten told the therapeutic recommended dose, which was $9 \mathrm{ml} . \mathrm{kg}^{-1}$. Animals of negative control group of each specie were orally treated with $10 \mathrm{ml} . \mathrm{kg}^{-1}$ of saline. Positive guinea-pigs citric acid-induced cough model was subcutaneously treated with $1 \mathrm{mg}^{-\mathrm{kg}^{-1}}$ of morphine. In the rats airway secretion model and japanese quails mucociliary transport rate, a positive control orally received $600 \mathrm{mg}^{\mathrm{kg}} \mathrm{kg}^{-1}$ of erdosteine. The results showed that both phytotherapic formulations were able to inhibit the guinea pig cough reflex induced by citric acid, being $36.4 \%$ of reduction in the Gripalplus Solução ${ }^{\circledR}$ group and $27.3 \%$ in the Melagrião ${ }^{\circledR}$ group. The rats airway secretion and the mucociliary transport rate were not significantly affected by the two phytotherapic formulations.

Keywords: antitussive, expectorant, phytotherapy, Gripalplus Solução ${ }^{\circledR}$, Melagrião (

\section{INTRODUÇÃO}

Alguns mecanismos do aparelho respiratório desempenham função de defesa e limpeza da árvore respiratória, que incluem o reflexo da tosse e o sistema mucociliar (Banner, 1986;
Kohrogi et al., 1989; Fontana et al., 2002; Pantaleo, 2002).

A tosse e o reflexo de broncoconstrição são manifestações comuns em doenças respiratórias (O’Connell, 2002). Os reflexos podem ser

Recebido em 18 de agosto de 2006

Aceito em 18 de maio de 2007

*Autor para correspondência (corresponding author)

E-mail: jmello@gabinete.ufrgs.br 
gerados por estimulação mecânica das vias aéreas, por inalantes irritantes ou por liberação local de mediadores, por ação nos receptores de adaptação rápida e nas terminações nervosas das fibras $\mathrm{C}$ presentes na laringe e mucosa traqueobrônquica, em humanos e em animais domésticos (Widdicombe, 2002).

O transporte mucociliar é outro mecanismo de defesa que serve para remover partículas inaladas da mucosa traqueobrônquica e contribui para a defesa pulmonar (Wanner, 1977). O sistema mucociliar é responsável pela movimentação dos fluidos (mucos), produzidos pelas células caliciformes e pelas glândulas brônquicas (Marriot et al., 1983; Marchioni et al., 1990; Górniak, 2002). Teoricamente, o mecanismo pelo qual há a interação do transporte mucociliar está diretamente relacionado com as alterações na quantidade e qualidade das secreções epiteliais (fluido periciliar e muco) e com as alterações na integridade anatômica e funcional dos cílios (Wanner, 1986)

As doenças relacionadas com o aparelho respiratório têm alta incidência em animais de estimação. $\mathrm{O}$ uso adequado de substâncias fitoterápicas na terapia instituída é o ponto-chave para o êxito do tratamento e para o bem-estar do paciente. Para o tratamento das doenças respiratórias, utilizam-se diversos fármacos, principalmente antitussígenos, expectorantes, antiasmáticos e tensoativos pulmonares, nem sempre com eficácia comprovada (Korolkovas, 1999).

O objetivo do trabalho foi avaliar duas formulações fitoterápicas em três modelos biológicos.

\section{MATERIAL E MÉTODOS}

Foram utilizados 40 ratos Wistar machos, com idade em torno de 100 dias e corporal de $326,6 \pm 4,9 \mathrm{~g}, 40$ cobaias albinas da linhagem Short Hair machos, com idade em torno de 100 dias e massa corporal de $693,4 \pm 14,2 \mathrm{~g}$, e 40 codornas japonesas (Coturnix coturnix japonica) machos, com idade em torno de 40 dias e massa corporal de $147,0 \pm 2,5 \mathrm{~g}$. Os animais, mantidos sob condições constantes de umidade, temperatura, $21^{\circ} \mathrm{C} \pm 2$, e fotoperíodo de 12 horas, claro das $9 \mathrm{~h}$ às $21 \mathrm{~h}$, foram alimentados com ração comercial e água ad libitum e aclimatados a esse regime durante 15 dias.

Foram utilizadas as formulações farmacêuticas que continham uma única planta ou extrato de plantas em associação e indicados como antitussígeno-expectorantes. Os fitoterápicos utilizados nos experimentos foram adquiridos de estabelecimentos farmacêuticos com o mesmo número de lote, data de fabricação e dentro do prazo de validade indicado pelo fabricante.

A composição quali-quantitativa de cada um deles é apresentada a seguir, conforme bula:

Formulação I ${ }^{1}$ - cada $150 \mathrm{ml}$ :

Extrato fluido de sabugueiro (Sambucus nigra).

Óleo de eucalipto (Eucalyptus globulus).

$0,1584 \mathrm{ml}$

Extrato fluido de agrião (Nasturtium officinale).

$0,2376 \mathrm{ml}$

Óleo de copaíba (Copaifera langsdorffi).

$0,0198 \mathrm{ml}$

Tintura de própolis

$0,2772 \mathrm{ml}$

Xarope com mel q.s.p

$150 \mathrm{ml}$

Formulação $\mathrm{II}^{2}$ - cada ml:

Alcoolatura de agrião (Nasturtium officinale).

$0,04666 \mathrm{ml}$

Extrato fluido de guaco (Mikania glomerata).

$0,00833 \mathrm{ml}$

Extrato fluido de polígala (Polygala sp.).

Solução concentrada de bálsamo de tolú (Myrospermum erytroxylon)....

$0,00833 \mathrm{ml}$

Acônito

$0,00800 \mathrm{ml}$

Excipiente q.s.p.

$0,00008 \mathrm{ml}$

$1,0 \mathrm{ml}$

\footnotetext{
${ }^{1}$ Gripaplus Solução® - Laboratório Pronabel Cosméticos e Perfumaria Ltda, Cascavel, PR (Lote 001/03 de julho de 2003).

${ }^{2}$ Melagrião® - Laboratório Catarinense. Herbarium, Joinvile, SC (Lote 5687 de janeiro de 2000).
} 
Nos protocolos experimentais com ratos e codornas, a erdosteína ${ }^{3}$ foi utilizada como fármaco com atividade mucolítica comprovada, possibilitando a constituição de grupos controlepositivo.

Para constituir o grupo-controle positivo no protocolo experimental de cobaia, o cloridrato de morfina foi usado como fármaco com atividade antitussígena comprovada (El-Hashim et al., 2004). Para avaliação quantitativa da secreção das vias aéreas, foram utilizados 10 ratos Wistar machos por grupo. Estes foram pesados e tratados por gavagem: os fitoterápicos e a dose utilizada corresponderam a 10 vezes a dose terapêutica recomendada pelo fabricante. Os grupos-controle negativo e positivo, constituídos por igual número de animais, foram tratados com solução fisiológica, $10 \mathrm{ml} . \mathrm{kg}^{-1}$, e erdosteína, $600 \mathrm{mg} \cdot \mathrm{kg}^{-1}$, respectivamente. Uma hora após, administrou-se fenolsulfoftaleína, $6 \mathrm{mg} \cdot \mathrm{kg}^{-1}$, por via endovenosa. Trinta minutos após a administração do corante, os animais foram sacrificados e deles obtido lavado broncoalveolar com $5 \mathrm{ml}$ de $\mathrm{NaHCO}_{3} 5 \%$. Centrifugado a 3000rpm, durante 30 minutos, o sobrenadante do lavado foi ajustado para pH 8.0. A absorbância foi medida em 558nm, e a concentração do corante foi calculada.

Para a avaliação do reflexo da tosse induzido pelo ácido cítrico em cobaias, foram utilizados 10 animais/grupo. Estes foram colocados na câmara de inalação de corpo inteiro e expostos ao ácido cítrico $5 \%$, durante cinco minutos, para registrar o número de episódios de tosse. Após três horas, os animais foram tratados e, uma hora depois, repetiu-se esse procedimento. O controle negativo foi tratado com solução fisiológica, $10 \mathrm{ml} . \mathrm{kg}^{-1}$, o controle positivo recebeu cloridrato de morfina, $1 \mathrm{mg} \cdot \mathrm{kg}^{-1}$, por via subcutânea, e ambos os grupos foram submetidos ao mesmo procedimento.

$\mathrm{Na}$ determinação da velocidade de transporte mucociliar na traquéia, as aves, dispostas em quatro grupos de 10, foram pesadas e tratadas. No grupo controle negativo foi administrada solução fisiológica, $10 \mathrm{ml} . \mathrm{kg}^{-1}$, e no controle positivo, erdosteína, $600 \mathrm{mg} \cdot \mathrm{kg}^{-1}$, ambos por gavagem.

\footnotetext{
${ }^{3}$ Flusten ${ }^{\circledR}$ cápsulas de $300 \mathrm{mg}$ - Laboratório Eurofarma Ltda, São Paulo, SP (Lote 002/02 de julho de 2002).
}

Após uma hora da administração, os animais foram anestesiados com uma associação de cloridrato de tiletamina e cloridrato de zolazepam, 50mg. $\mathrm{kg}^{-1} \mathrm{IM}$. A traquéia foi incisada longitudinalmente $(5 \mathrm{~mm})$, e as aves submetidas à microscópia estereoscópica. Uma partícula do marcador foi introduzida na camada mucosa da traquéia, e registrado o tempo decorrente da sua movimentação em cinco milímetros. $\mathrm{O}$ movimento mucociliar foi avaliado a cada 10 minutos durante 120 minutos consecutivos, e a velocidade foi calculada.

Os resultados foram apresentados como média \pm EPM. A metodologia utilizada para análise estatística incluiu análise de variância de uma via, análise de variância de medidas repetidas e teste de Bonferroni (Zar, 1999; Callegari-Jacques, 2004). O nível de significância adotado foi $\mathrm{P}<0,05$.

\section{RESULTADOS}

Na Fig. 1 mostra-se a concentração de corante $(\mu \mathrm{g} / \mathrm{ml})$ obtida no lavado broncoalveolar de ratos de acordo com os grupos. Houve diferença estatisticamente significativa entre os grupos $(\mathrm{P}=0,006)$. $\mathrm{O}$ grupo controle-positivo foi estatisticamente diferente do grupo-controle negativo e do grupo tratado com a formulação I.

Na Tab. 1 apresenta-se o número de eventos de tosse dos animais expostos a aerossóis de solução aquosa de ácido cítrico $5 \%$ após as duas exposições seqüenciais.

Houve diferença entre os grupos quanto ao percentual de significativa redução dos eventos de tosse $(\mathrm{P}<0,001)$. Pelo teste Bonferroni, o grupo-controle positivo e os grupos das formulações I e II foram estatisticamente diferentes do grupo-controle negativo.

Na Fig. 2 mostra-se a velocidade do transporte mucociliar aferida por meio da adição de uma partícula marcadora na traquéia das codornas. Houve diferença significativa entre os grupos em alguns tempos medidos $(\mathrm{P}=0,025)$. Pelo teste Bonferroni, o grupo-controle positivo foi diferente do grupo-controle negativo nas medidas realizadas aos 80, 90, 100, 110 e 120 minutos. 


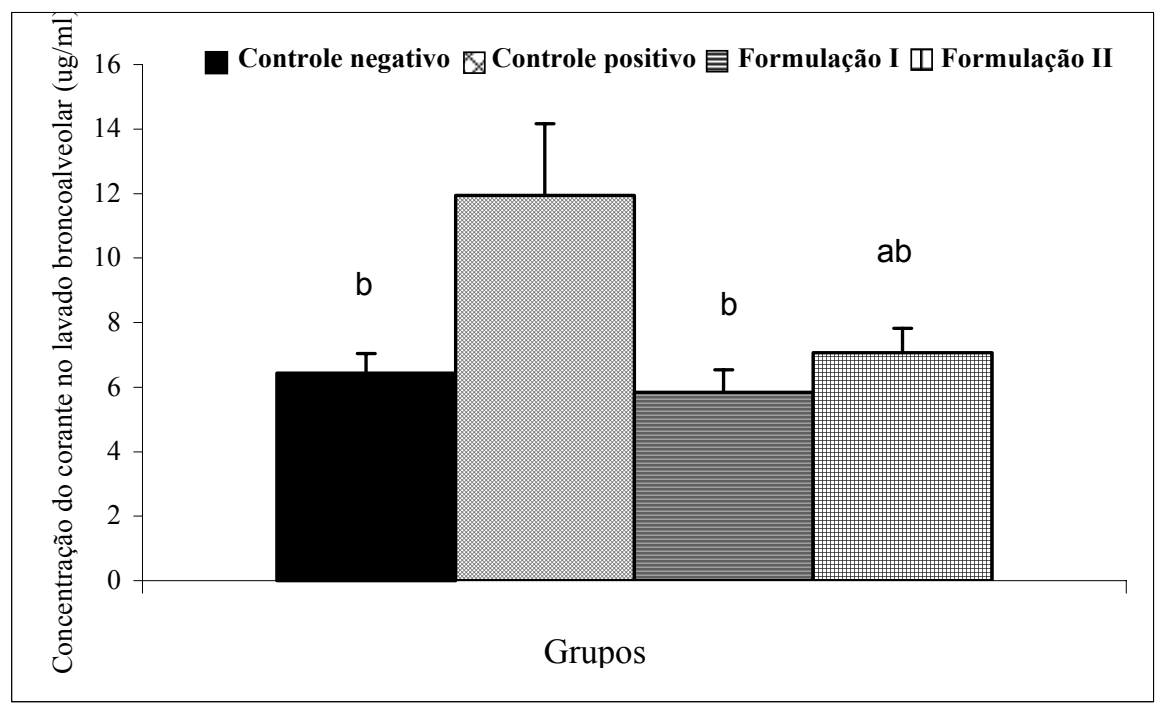

Figura 1. Concentração de fenolsulfoftaleína $(\mu \mathrm{g} / \mathrm{ml})$ obtida no lavado broncoalveolar de ratos de acordo com o grupos. Os valores representam as médias, e as barras verticais representam os erros-padrão dos médios $(\mathrm{n}=10$ animais/grupo). Letras diferentes indicam diferença entre grupos $(\mathrm{P}<0,05)$.

Tabela 1. Número e percentual de modificação dos eventos de tosse apresentados pelos animais expostos a aerossóis de solução aquosa de ácido cítrico $5 \%$ após duas exposições seqüenciais: médias e erros-padrão das médias de acordo com os grupos ( $\mathrm{n}=10$ animais/grupo)

\begin{tabular}{lccc}
\hline Grupo & Primeira exposição & Segunda exposição & Modificação (\%) \\
\hline Formulação I & $11,0 \pm 2,67$ & $8,8 \pm 2,62$ & $-36,4 \pm 18,87^{*}$ \\
Formulação II & $10,3 \pm 1,68$ & $7,4 \pm 1,50$ & $-27,3 \pm 15,73^{*}$ \\
Controle negativo & $11,3 \pm 1,13$ & $15,4 \pm 2,48$ & $+20,6 \pm 8,83$ \\
Controle positivo & $17,9 \pm 2,47$ & $3,2 \pm 1,00$ & $-84,2 \pm 4,39^{*}$ \\
\hline
\end{tabular}

*Diferença significativa $(\mathrm{P}<0,05)$.

Controle negativo $\oslash$ Controle positivo 国 Formulação I $\boxplus$ Formulação II

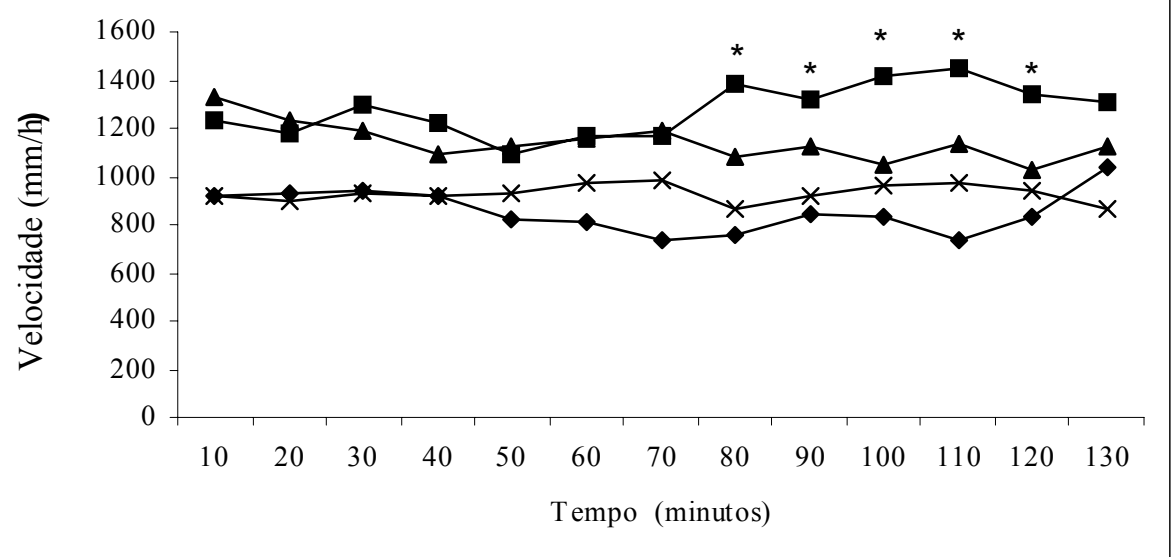

Figura 2. Velocidade do transporte mucociliar $(\mathrm{mm} / \mathrm{h})$ aferida por meio da adição de uma partícula marcadora (carvão ativado) na traquéia das codornas de acordo com os grupos. Os valores representam as médias dos grupos em cada tempo medido. *Diferença significativa em relação ao grupo controle negativo $(\mathrm{P}<0,05)$. 


\section{DISCUSSÃO}

Em muitos mecanismos relacionados com a expectoração, os expectorantes atuam por redução da viscosidade do muco, promoção da secreção dos fluidos do trato respiratório e normalização do muco traqueal (Hosoe et al., 1999). Os dois fitoterápicos estudados neste trabalho foram avaliados quanto a sua capacidade de modular a quantidade de secreção das vias aéreas, suprimir eventos de tosse e modificar a velocidade de transporte mucociliar. Para tanto, as duas apresentações fitoterápicas foram avaliadas em cada modelo experimental proposto.

Quando testado quanto à avaliação da secreção das vias aéreas em ratos, nenhum deles apresentou a capacidade de alterar a quantidade de secreção produzida pela árvore traqueobrônquica. Embora tenha havido aumento da concentração do corante no lavado broncoalveolar dos ratos tratados com a formulação II, os resultados não permitiram concluir pela eficácia desse fitoterápico.

A propriedade de liquefazer as secreções das vias aéreas é uma forma de facilitar a sua eliminação e auxiliar o organismo nos seus mecanismos defensivos da árvore respiratória (Korolkovas, 1999), mas existem outros mecanismos para que as ações defensivas sejam estimuladas e facilitadas. A secreção do trato respiratório tem papel importante na expectoração, pois as características reológicas estão intimamente relacionadas com a velocidade de transporte mucociliar (Hosoe et al., 1999).

Os fármacos que aumentam o volume de secreções podem ser denominados fármacos com atividade secretagoga e, quando uma substância apresenta a propriedade de aumentar, de forma consistente, a secreção da árvore respiratória, esse fato pode representar uma vantagem na terapêutica clínica (Disse e Ziegler, 1987).

$\mathrm{Na}$ experimentação dos fitoterápicos no modelo de avaliação do reflexo da tosse induzido pelo ácido cítrico em cobaias, diferente do ocorrido no modelo anterior, os fitoterápicos apresentaram determinadas propriedades antitussígenas.

O percentual de redução dos eventos de tosse apresentados pelos animais de cada grupo permite fazer avaliações com relação à potência individual de cada fitoterápico, sendo o mais potente aquele que produziu maior percentual de redução da tosse na exposição desafio. O mecanismo de ação que justifique o efeito antitussígeno pode ser atribuído a cada um dos constituintes presentes nas plantas ou à associação das constituintes. Devido à composição dos fitoterápicos, admite-se que esses atuam nos receptores periféricos da tosse e não por meio de mecanismos centrais, conforme o ocorrido no grupo-controle positivo (Chung e Chang, 2002). Dessa forma, a inibição da tosse apresentada nos grupos tratados ocorreu pela ação nas vias periféricas (Carr e Ellis, 2002). Essa ação ocorre em virtude da redução na transmissão dos impulsos originários, principalmente dos receptores de adaptação rápida e dos receptores fibras $\mathrm{C}$ (Ricciardolo, 2001; Ziment e O’Connell, 2002).

Quando se avaliaram os fitoterápicos quanto ao modelo de determinação da velocidade de transporte mucociliar em codornas, observou-se que nenhum deles apresentou eficácia, quando comparado com o grupo-controle negativo.

Substâncias que aumentam a velocidade do transporte mucociliar têm grande aplicabilidade terapêutica (Miyata et al., 1998; Korolkovas, 1999) e todas as plantas que compõem as duas formulações fitoterápicas estudadas têm indicações terapêuticas para o tratamento das patologias do aparelho respiratório (Oliveira et al., 1993) e apresentam ação sobre este, razão pela qual podem ser denominadas antitussígenas periféricas que atuam nos receptores da tosse localizados no epitélio das vias respiratórias (Korolkovas, 1999).

A adição dos hidratantes às formulações fitoterápicas utilizadas provavelmente conferiu a elas as propriedades antitussígenas periféricas, além de ação resultante dos constituintes químicos das plantas individualmente ou em associação.

As plantas usadas apresentam constituintes químicos tais como: óleos essenciais, taninos, terpenóides, flavonóides e saponinas, dentre outros. A associação desses constituintes ou alguns deles, especificamente, apresentam propriedades antiinflamatórias que provavelmente são responsáveis por parte das ações desses 
fitoterápicos. Essa ação se expressa de forma consistente no modelo de avaliação do reflexo da tosse induzido pelo ácido cítrico.

É possível que a retirada de uma ou mais plantas das formulações testadas acarrete em redução na eficácia do preparado. Assim, a exclusão de alguma delas nas associações pode acarretar na redução da eficácia e na necessidade de aumentar a concentração das plantas remanescentes no preparado e, desse modo, alterar os resultados relacionados à toxicidade do produto.

\section{CONCLUSÃO}

O estudo feito neste trabalho sugere que as formulações fitoterápicas têm eficácia terapêutica como antitussígeno.

\section{REFERÊNCIAS BIBLIOGRÁFICAS}

BANNER, A.S. Cough: physiology, evaluation and treatment. Lung, v.164, p.79-92, 1986

CALLEGARI-JACQUES, S.M. Bioestatística: princípios e aplicações. São Paulo: Ed. Artmed Editora, 2004. 255p.

CARR, M.J.; ELLIS, J.L. The study of airway primary afferent neuron excitability. Curr. Opin. Pharmacol., v.2, p.216-219, 2002.

CHUNG, K.F.; CHANG, A.B. Therapy for cough: active agents. Pulm. Pharmacol. Therap., v.15, p.335$338,2002$.

DISSE，B.G.; ZIEGLER，H.W. Pharmacodynamic mechanism and therapeutic activity of ambroxol in animal experiments. Respiration, v.51, p.15-22, 1987.

EL-HASHIM, A. Z.; WYSS, D.; LEWIS, C. Effect of a novel NK1 receptor selective antagonist (NKP608) on citric acid induced cough and airway obstruction. Pulm. Pharmacol. Therap., v.17, p.11-18, 2004.

FONTANA, G.A.; LAVORINI, F.; PISTOLESI, M. Water aerosols and cough. Pulm. Pharmacol. Therap. v. 15, p. $205-211,2002$

GÓRNIAK, S.L. Medicamentos com ação no sistema respiratório. In: SPINOSA, H.S.; GÓRNIAK, S.L.; BERNARDI, M.M. (Eds). Farmacologia aplicada à medicina veterinária. 3.ed. Rio de Janeiro:Guanabara Koogan, 2002. p.158-166.

HOSOE, H.; KAISE, T.; ISOHAMA, T.H. et al. Mucolytic and antitussive effects of erdosteine. $J$. Pharmac. Pharmacol., v.51, p.959-966, 1999.
KOHROGI, H.; GRAF, P.D.; SEKIZAWA, K. et al. Recombinant human enkephalinase (neutral endopeptidase) prevents cough induced by tachykinins in awake guinea pigs. J. Clin. Invest., v.82, p.20632068, 1989.

KOROLKOVAS, A. Dicionário terapêutico Guanabara. São Paulo: Guanabara Koogan, 1999. $721 \mathrm{p}$.

LAPPONI, J. C. Estatística usando o Excel. 2.ed. São Paulo: Lapponi Treinamento e Editora, 2000. 451p.

MARCHIONI, C.F.; MORETTI, M.; MURATORI, M. et al. Effects of erdosteine on sputum biochemical and rheological properties: pharmacokinetics in chronic obstructive lung disease. Lung, v.168, p.285293, 1990.

MARRIOT, C.; READMAN, A.S.; BARRETT-BEE, $\mathrm{K}$. The effect of some drugs on the biophysical and biochemical properties of pig tracheal mucus. Eur. J. Respir. Dis., v.64, p.441-443, 1983.

MIYATA, T.; KAI, H.; ISOHAMA, Y. et al. Current opinion of muco active drug research: strategies and problems. Eur. Respir. J., v.11, p.480-491, 1998.

O'CONNELL, F. Central pathways for cough in man - unanswered questions. Pulm. Pharmacol. Therap., v.15, p.295-301, 2002.

OLIVEIRA, F.; SAITO, M.L.; GARCIA, L.O. Caracterização cromatográfica em camada delgada do extrato fluido de guaco - Mikania glomerata Sprengel. LECTA-USF., v.11, p.43-56, 1993.

PANTALEO, T.; BONGIANNI, F.; MUTOLO, D. Central nervous mechanisms of cough. Pulm. Pharmacol. Therap., v.15, p.227-233, 2002.

RICCIARDOLO, F.L.M. Mechanisms of citric acidinduced bronchoconstriction. Am. J. Med., v.111, p.18s-24s, 2001.

WANNER, A. Clinical aspects of mucociliary transport. Am. Rev. Resp. Dis., v.116, p.73-125, 1977.

WANNER, A. Effect of ibatropium bromide on airway mucociliary function. Am. J. Med., v.81, p.2327, 1986.

WIDDICOMBE, J. Neuroregulation of cough: implications for drug therapy. Curr. Opin. Pharmacol., v.2, p.256-263, 2002.

ZAR, J.H. Biostatistical analysis. 4.ed. New Jersey: Prentice Hall, 1999. 123p.

ZIMENT, I.; O'CONNELL, F. Summary: clinical needs for cough therapy. Pulm. Pharmacol. Therap., v.15, p.293-294, 2002. 